\title{
HAND GESTURE BASED WHEEL CHAIR WITH OBSTACLE DETECTION,WIRELESS \& GPS TECHNOLOGY USING BLUETOOTH
}

\author{
Saish S. Shinde ${ }^{1}$, Sayli R. Kadam ${ }^{2}$, Madhavi R. Kadam ${ }^{3}$, Ronel Paul ${ }^{4}$ \\ ${ }^{1}$ Saish S. Shinde,Electronics, K.J.S.I.E.I.T, Maharashtra, India \\ ${ }^{2}$ Sayli R. Kadam, Electronics, K.J.S.I.E.I.T., Maharashtra, India \\ ${ }^{3}$ Madhavi R. Kadam, Electronics, V.I.T., Maharashtra, India \\ ${ }^{4}$ Ronel Paul, Electronics And Telecommunication, S.F.I.T, Maharashtra ,India
}

\begin{abstract}
This paper is to develop a wheelchair control which is useful to the physically disabled person with his hand movement or his hand gesture recognition using Acceleration technology. Various Projects have been undertaken previously to develop automatic wheelchair using various technology like button control, Retina controlled. But none have such combined features like Navigation, Dark Room Sensor, Automatic messaging, Obstacle detector. This project will make the user life more comfortable and more independent and is also cost effective and requires low maintenance. Tremendous leaps have been made in the field of wheelchair technology. However, even these significant advances haven't been able to help quadriplegics navigate wheelchair unassisted. It is wheelchair which can be controlled by simple hand gestures. It employs a sensor which controls the wheelchair hand gestures made by the user and interprets the motion intended by user and moves accordingly. Microcontroller controls the wheelchair directions like LEFT, RIGHT, FRONT, and BACK. The aim of this paper is to implement wheelchair direction control with hand gesture reorganization. The features included in this project are, We are using touch plate sensors to control wheelchair movement, Obstacle detection with the message on mobile via Bluetooth and deviation from the obstacle, Panic Switch with the alert message to the near ones, Illumination of the LEDs in the dark places, Navigation through Google maps.
\end{abstract}

Keywords: IR led, Obstacle sensor, Diode etc..

\section{INTRODUCTION}

Our project aims at developing a wheelchair for disabled people and help them as much as possible. As described in the features, apart from hand gesture controlled wheelchair, there are some additional features which we have added to make it more helpful like-

1) Obstacle sensor- it senses the nearby obstacles (upto the range of $5-6 \mathrm{~cm}$ in case of our prototype). It does not stop instead it deviates from the obstacle. If it senses obstacle in the left direction, a voice message will be received and the wheelchair will automatically move towards the right direction. This will not only make the disabled person aware of the obstacles instead it will help him to get to the right path.

2) Dark light sensors- these sensors are meant for making the path visible in a dark place. The disabled person can see the hurdles coming in the path and avoid them with the help of this feature.

3) GPS based navigation- it is used for guiding the direction to the destination just like google maps.

4) Panic switch- this feature is useful for the situations where the handicapped person needs immediate attention. The person can press this switch and an emergency message would be sent to his dear ones via Bluetooth module. They can be aware of the disabled person's location and can help him out in this panic situation.

\section{Methodology}

\subsection{Obstacle Detector:}

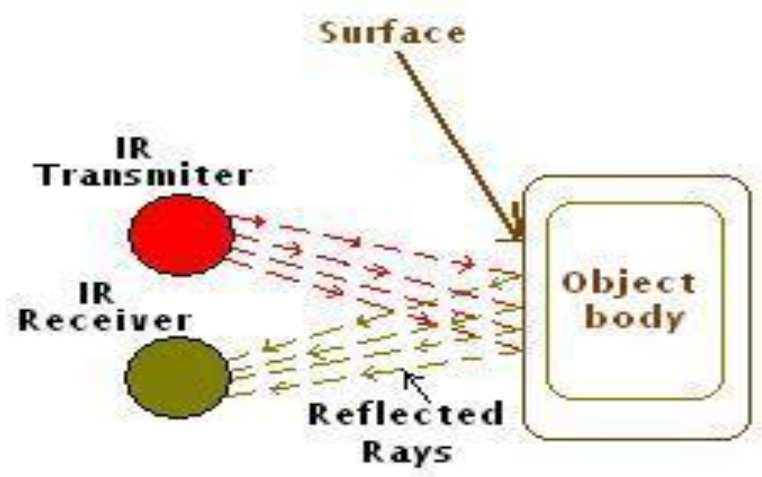

Fig -1:Mechanism of obstacle detector

It consists of three major components. The first is an InfraRed (IR) transmitter (usually an IR LED), the second is a TSOP (an Infra-Red receiver) and third IC 555.

The main difference between LED and IR LED is that IR LED emits Infrared Radiations, which we cannot see by our naked eyes. TSOP requires the incoming data to be modulated at a particular frequency and would ignore any other signals. It is also immune to ambient IR light. They are available for different carrier frequencies from $32 \mathrm{kHz}$ to 42 $\mathrm{kHz}$. 
IC555 is a Timer IC using which we are generating sequence of square wave pulses. It is configured in Astable mode to generate the square wave signal at particular frequency.

\section{Algorithm:}

- IC 555 generates square wave and give it to IR Transmitter which transmit wave

- IR Receiver waits for reflected rays for some duration

- If no obstacle is present, no reflection and transmitter again transmit the wave

- If obstacle is present wave reflected to receiver and following action takes place

Obstacle on front $\rightarrow$ Stop

Obstacle on right $\rightarrow$ Move to left

Obstacle on left $\rightarrow$ Move to Right

\subsection{MOTOR:}

In a simple 2-pole DC electric motor, as shown above (here red (right side) represents a magnet or winding with a "North" polarization, while green (left side) represents a magnet or winding with a "South" polarization).

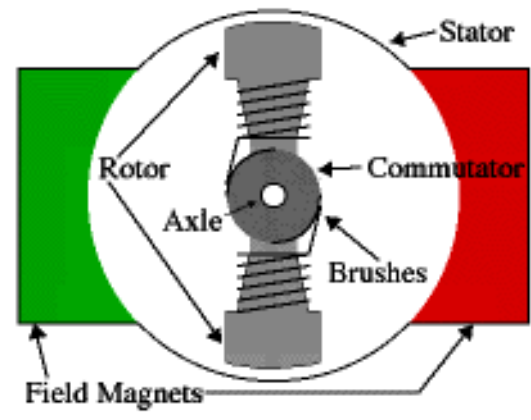

Fig -2: DC motor

Every DC motor has six basic parts axle, rotor (a.k.a., armature), stator, commutator, field magnet(s), and brushes. In most common DC motors (and all that Beamers will see), the external magnetic field is produced by high-strength permanent magnets.

The stator is the stationary part of the motor this includes the motor casing, as well as two or more permanent magnet pole pieces. The rotor (together with the axle and attached commutator) rotates with respect to the stator. The rotor consists of windings (generally on a core), the windings being electrically connected to the commutator. The above diagram shows a common motor layout with the rotor inside the stator (field) magnets.

The geometry of the brushes, commutator contacts, and rotor windings are such that when power is applied, the polarities of the energized winding and the stator magnet(s) are misaligned, and the rotor will rotate until it is almost aligned with the stator's field magnets. As the rotor reaches alignment, the brushes move to the next commutator contacts, and energize the next winding. Given our example two-pole motor, the rotation reverses the direction of current through the rotor winding, leading to a "flip" of the rotor's magnetic field, driving it to continue rotating.

\subsection{Bluetooth Module:}

This technology achieves its goal by embedding tiny, inexpensive, short-range transceivers into the electronic devices that are available today. The radio operates on the globally-available unlicensed radio band, $2.45 \mathrm{GHz}$ (meaning there will be no hindrance for international travelers using Bluetooth-enabled equipment.), and supports data speeds of up to $721 \mathrm{Kbps}$, as well as three voice channels. The bluetooth modules can be either built into electronic devices or used as an adaptor. For instance in a PC they can be built in as a PC card or externally attached via the USB port.

Each device has a unique 48-bit address from the IEEE 802 standard. Connections can be point-to-point or multipoint. The maximum range is 10 meters but can be extended to 100 meters by increasing the power. Bluetooth devices are protected from radio interference by changing their frequencies arbitrarily up to a maximum of 1600 times a second, a technique known as frequency hopping. They also use three different but complimentary error correction schemes. Built-in encryption and verification is provided.

Moreover, Bluetooth devices won't drain precious battery life. The Bluetooth specification targets power consumption of the device from a "hold" mode consuming 30 micro amps to the active transmitting range of 8-30 milliamps (or less than $1 / 10$ th of a watt). The radio chip consumes only $0.3 \mathrm{~mA}$ in standby mode, which is less than $3 \%$ of the power used by a standard mobile phone. The chips also have excellent power-saving features, as they will automatically shift to a low-power mode as soon as traffic volume lessens or stops.

\subsection{Microcontroller (89S52):}

The AT89S52 is a low-power, high-performance CMOS 8bit microcontroller with $8 \mathrm{~K}$ bytes of in-system programmable Flash memory. The device is manufactured using Atmel's high-density nonvolatile memory technology and is compatible with the indus-try-standard 80C51 instruction set and pinout.

By combining a versatile 8-bit CPU with in-system programmable Flash on a monolithic chip, the Atmel AT89S52 is a powerful microcontroller which provides a highly-flexible and cost-effective solution to many embedded control applications. The AT89S52 provides the following standard features: $8 \mathrm{~K}$ bytes of Flash, 256 bytes of RAM, 32 I/O lines, Watchdog timer, two data pointers, three 16-bit timer/counters, a six-vector two-level interrupt architecture, a full duplex serial port, on-chip oscillator, and clock circuitry. In addition, the AT89S52 is designed with static logic for operation down to zero frequency and supports two software selectable power saving modes.

\subsection{L293D Motor Driver IC:}

L293D is a typical Motor driver or Motor Driver IC which allows DC motor to drive on either direction. L293D is a 16pin IC which can control a set of two DC motors simultaneously in any direction. It means that you can control two DC motor with a single L293D IC. The 1293d can drive small and quiet big motors as well. It works on the 
concept of H-bridge. H-bridge is a circuit which allows the voltage to be flown in either direction. H-bridge IC is ideal for driving a DC motor. In a single $1293 \mathrm{~d}$ chip there two hBridge circuit inside the IC which can rotate two dc motor independently.

Due its size it is very much used in robotic application for controlling DC motors. Given below is the pin diagram of a L293D motor controller. There are two Enable pins on 1293d. Pin 1 and pin 9, for being able to drive the motor, the pin 1 and 9 need to be high. For driving the motor with left $\mathrm{H}$-bridge you need to enable pin 1 to high. And for right $\mathrm{H}-$ Bridge you need to make the pin 9 to high. If anyone of the either pin1 or pin9 goes low then the motor in the corresponding section will suspend working.

\section{L293D Logic Table:}

- $\quad$ Pin2 =Logic 1 and Pin7=Logic $0 \mid$ Clockwise Direction.

- $\operatorname{Pin} 2=$ Logic 0 and Pin7=Logic 1 | Anticlockwise Direction.

- $\quad$ Pin2= Logic 0 and Pin7= Logic $0 \mid$ Idle [No rotation] [Hi-Impedance state].

- $\quad \operatorname{Pin} 2=\operatorname{Logic} 1$ and Pin7 = Logic $1 \mid$ Idle [No rotation].

\section{MECHANISM OF DARK LIGHT SENSOR:}

There are many ways to realize remote object detection. The simplest is just IR Light Emitting Diode (later LED) and phototransistor. IR LED emits light with wavelength approximately 850 nanometers. Light reaches obstacle and reflects back. There it is picked up with phototransistor. Voltage in measurement point changes (fig. 1), and this change is proportional to picked up light intensity.

Comparator or ADC might be used to convert it to digital form and decide, if there is an obstacle in front of us. For example if 5 Volts supply is used: 4.5 Volts mean obstacle is far, and 1 Volt means obstacle is close (as phototransistor current flows, voltage dropout on resistor grows according to Ohm's law, and voltage in point of measurement falls).

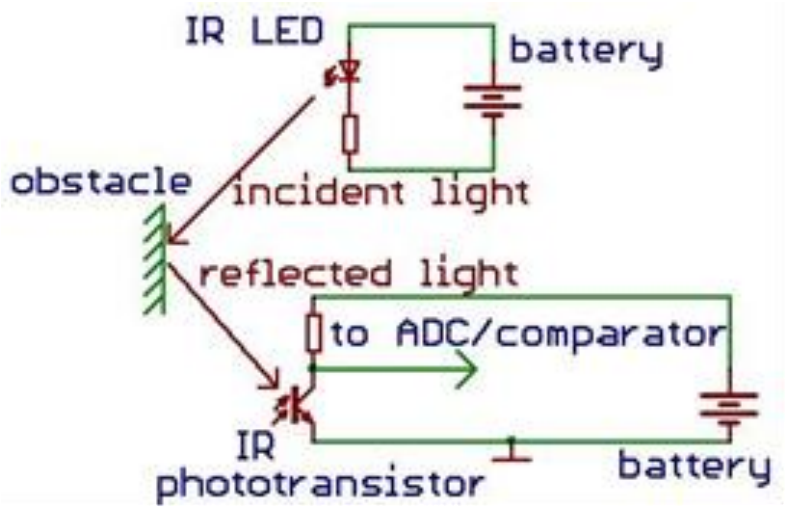

Fig -3: Mechanism of dark light sensing.

This schematic has big drawback. First, the Sun is powerful IR source, so there will be false readings on sunlight. Also any light bulb emits IR light intensely, so false readings again.

\section{CIRCUIT LAYOUT}

A microcontroller usually comprises of a CPU, ROM, RAM and $\mathrm{I} / \mathrm{O}$ ports, built within it to execute a single and dedicated task. It has $8 \mathrm{kB}$ Flash and 256 bytes of data RAM32 I/O lines. Microcontrollers are designed for embedded applications, in contrast the microprocessors used in personal computers or other general purpose applications.

Component List:-

\begin{tabular}{|l|l|l|l|}
\hline $\begin{array}{l}\text { Sr } \\
\text { no. }\end{array}$ & $\begin{array}{l}\text { Abbreviati- } \\
\text { on }\end{array}$ & Component & Value \\
\hline 1 & D1 & DIODE & $1 \mathrm{~N} 4007$ \\
\hline 2 & SW1 & SWITCH & - \\
\hline 3 & C1,C5 & CAPACITOR & $10 \mathrm{Uf}$ \\
\hline 4 & C2 & CAPACITOR & $100 \mathrm{uF}$ \\
\hline 5 & C3,C4 & CAPACITOR & $33 \mathrm{Pf}$ \\
\hline 6 & X1 & CRYSTAL & $11.0592 \mathrm{MHz}$ \\
\hline 7 & RT1 & REGULATOR & 7805 \\
\hline 8 & IC1 & $\begin{array}{l}\text { P0 } \\
\text { SOCKET(89S52) }\end{array}$ & - \\
\hline 9 & L1 & LED & $3 \mathrm{~mm}$ \\
\hline 10 & R1 & RESISTOR & $270 \Omega$ \\
\hline 11 & R5 & RESISTOR & $8.2 \mathrm{~K} \Omega$ \\
\hline 12 & RST1 & RESET SWITCH & - \\
\hline 13 & B1 & BUG STRIP & - \\
\hline 14 & $89 S 52$ & MICROCONTROLLER & - \\
\hline
\end{tabular}

Chart-1: List of components for TSOP.

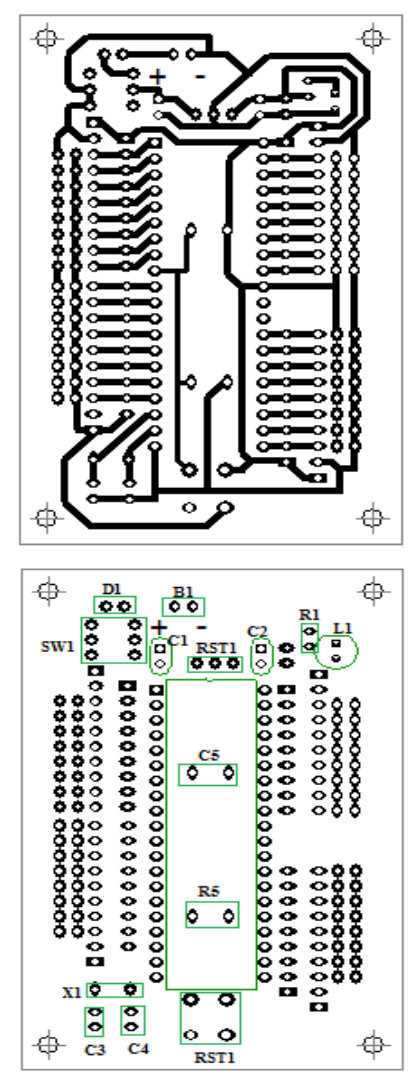

Fig -4: Layout of Components 
The TSOP 1738 is a member of IR remote control receiver series. The output of TSOP is active low and it gives $+5 \mathrm{~V}$ in off state. When IR waves, from a source, with a center frequency of $38 \mathrm{kHz}$ incident on it, its output goes low.

The microcontroller is configured as follow to move the wheelchair:

\begin{tabular}{|l|l|l|l|l|}
\hline PARAMETER & PORT & PORT & PORT & PORT \\
& 0.0 & 0.1 & 0.2 & 0.3 \\
\hline FORWARD & CLEAR & SET & CLEAR & SET \\
\hline BACKWARD & CLEAR & CLEAR & SET & CLEAR \\
\hline LEFT & CLEAR & CLEAR & CLEAR & SET \\
\hline RIGHT & CLEAR & SET & CLEAR & CLEAR \\
\hline STOP & CLEAR & CLEAR & CLEAR & CLEAR \\
\hline
\end{tabular}

Chart-2: Configuration of ports for movement.

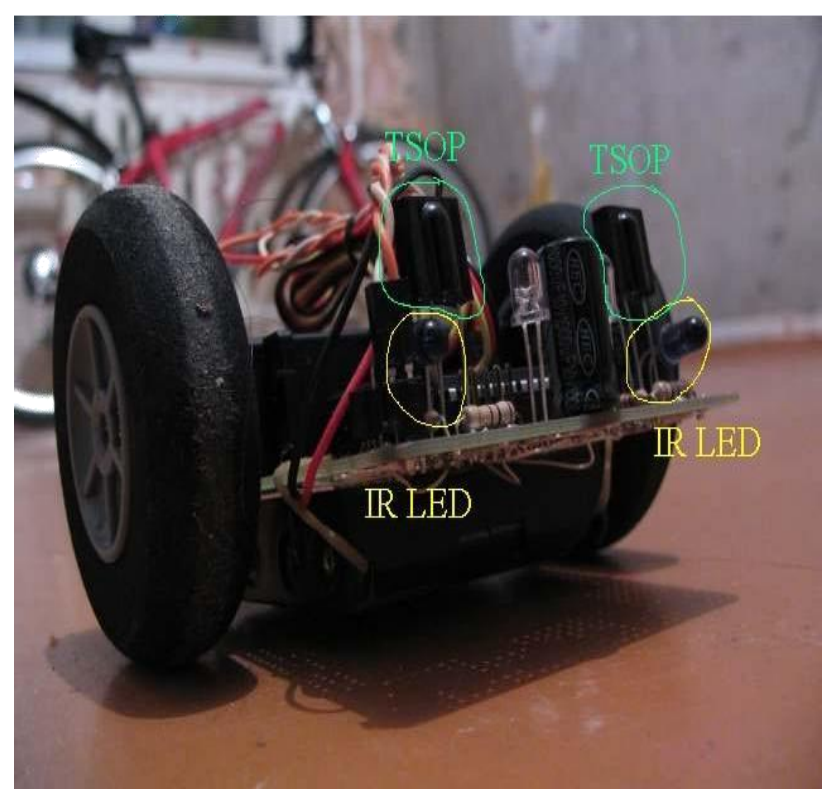

Fig -5: Overall wheel chair system.

\section{CONCLUSIONS}

This project has been made for handicapped and disable people who are not able to live like a common man in this environment. In this project we propose a gesture-based interface for connection and control of multiple devices in the environment. With simple selection and pointing gesture, users can easily control connections between multiple devices as well as manage information or data between them.

Wheelchair input control selection is very important for people with disabilities as the comfort and safety of the operation directly influences their quality of life and social ability. The results from this study revealed that the wireless hand glove commander developed in this study, although not absolutely superior to the joystick controlled method, should still provide better option for those people unsuited to traditional input devices.

\section{REFERENCES}

[1] Yoshinori Kunotl, Teruhisa Murashimat ,Nobutaka Shimadat and Yoshiaki Shirait Interactive Gesture Interface for Intelligent Wheelchairs, ,

[2] Donald P. Massa, Choosing an Ultrasonic Sensor for Proximity or Distance

Measurement Part 1: Acoustic Considerations,,.

[3] S. Fioretti, T. Leo, and S. Longhi, _A Navigation System for Increasing the

Autonomy and the Security of Powered Wheelchairs, IEEE Transactions On Rehabilitation Engineering, Vol. 8, No. 4, December 2000.

[4] Rajesh Kannan Megalingam, Ramesh Nammily Nair, Automated Voice based Home Navigation System for the Elderly and the Physically Challenged\| Feb. 13 16, 2011.

\section{Biographies}

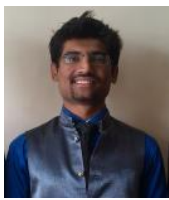

Saish S. Shinde.

$\mathrm{BE}$ (Electronics)

K. J. Somaiya Institute of Engineering \& I.T.

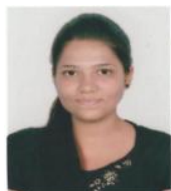

Sayli R. Kadam

BE(Electronics)

K. J. Somaiya Institute of Engineering \& I.T.

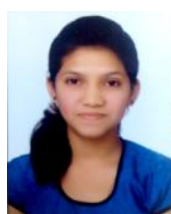

Madhavi R. Kadam

BE(Electronics)

Vidyalankar Institute of

Technology

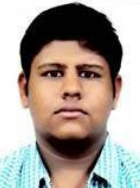

\section{Ronel Paul}

BE (Electronics And Telecommunication)

St. Francis Institute of

Technology 